\title{
érès
}

\section{Préparation à la naissance}

\author{
Michel Briex
}

DANS SPIRALE 2008/3 (N²7), PAGES 181 À 183

ÉDITIONS ÉRÈS

ISSN 1278-4699

ISBN 9782749209326

DOI 10.3917/spi.047.0181

Découvrir le sommaire de ce numéro, suivre la revue par email, s'abonner... Flashez ce QR Code pour accéder à la page de ce numéro sur Cairn.info.

Distribution électronique Cairn.info pour Érès.

La reproduction ou représentation de cet article, notamment par photocopie, n'est autorisée que dans les limites des conditions générales d'utilisation du site ou, le cas échéant, des conditions générales de la licence souscrite par votre établissement. Toute autre reproduction ou représentation, en tout ou partie, sous quelque forme et de quelque manière que ce soit, est interdite sauf accord préalable et écrit de l'éditeur, en dehors des cas prévus par la législation en vigueur en France. Il est précisé que son stockage dans une base de données est également interdit. 


\section{Préparation à la naissance}

La préparation à la naissance s'est développée dans notre pays avec la prise en charge par l'assurance maladie des cours de PPO (préparation psychoprophylactique obstétricale) en 1956 sous l'impulsion du docteur Lamaze faisant partie d'un courant de l'époque nommé " accouchement sans douleur " qui a pris naissance en Angleterre et en Union soviétique à la fin des années 1930 et au début des années 1940.

La méthode du docteur Lamaze et de son équipe était, pour faire simple, basée sur des techniques respiratoires dont la plus célèbre est celle dite $d u$ " petit chien " pendant les contractions et l'expulsion.

Ces techniques destinées principalement et initialement à réduire le vécu des douleurs de l'accouchement sont rapidement passées au second plan pour la gestion de la douleur depuis la mise à disposition d'une technique médicale bien plus efficace qui est la péridurale. En France autour de 50 à $60 \%$ des parturientes accouchent sous péridurale et dans certaines maternités jusqu'à près de $90 \%$.

Si l'utilité de ces cours de préparation ne fait aucun doute sur le vécu de la grossesse, de l'accouchement, le choix des possibilités d'accompagnement de la naissance, l'apprentissage de la gestion des postures pendant le travail et l'expulsion du bébé, la préparation à un meilleur déroulement, l'allaitement maternel et d'autres points importants pour les parturientes, on peut se questionner sur ses avantages actuels sur le ressenti de la douleur du travail ou la gestion des sensations de l'accouchement.

Un travail de mémoire présenté récemment (Audrey Laurent, "La douleur de l'accouchement ", université Paul-Sabatier, 2006-2007) s'est appuyé sur une enquête faite sur le site Gyneweb (http://www. gyneweb.fr) venant confirmer des notions déjà décrites auparavant. L'étude a porté sur 795 réponses dont 510 se sont avérées exploitables.

D'abord les attentes des femmes enceintes interrogées portent majoritairement sur la douleur, que ce soit sur les capacités à la gérer, les explications sur les techniques de gestion et les possibilités d'accom-

Michel Briex, CH Libourne, gynécologie, obstétrique. 
pagnement. Obtenir des explications sur la péridurale leur apparaît tout aussi primordial avec un nombre identique de réponses.

L'attente d'un soutien affectif, d'un espace de parole, d'échange, et la possibilité de comprendre sur le plan physiologique ce qui se passe et ce que l'on ressent sont aussi mises en avant par les parturientes.

Dans une autre étude un peu plus ancienne (F. Cornillot, JTA, 1999) on s'est intéressé a posteriori au ressenti des patientes en fonction ou non du suivi de cours de la préparation à la naissance.

Sur cette étude $36 \%$ des parturientes avaient suivi ces cours avec une bonne assiduité, $61 \%$ avaient bénéficié d'une péridurale (76 \% chez les primipares et $49 \%$ chez les multipares).

Si I'on comparait cette population ayant suivi les cours de préparation aux autres accouchées, cette étude montrait qu'en salle de naissance ces patientes ressentent plus intensément leurs contractions et que globalement la douleur ressentie pendant le travail était supérieure à ce qu'elles avaient imaginé faisant conclure à l'auteure : " La préparation à la naissance qui a sa place à part entière dans la prise en charge de la douleur en obstétrique reste néanmoins, telle qu'elle est pratiquée actuellement dans notre service, une méthode bien limitée dans le soulagement de la douleur. "

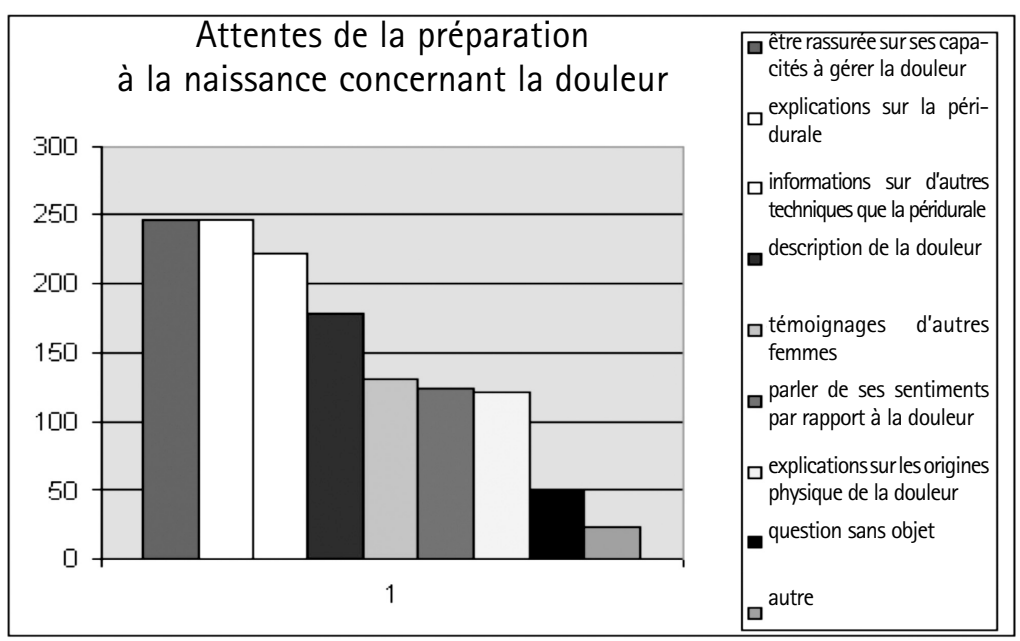

Des études portant sur des méthodes spécifiques ont elles aussi comparé le vécu de la douleur avec une préparation portant sur l'hypnose, la sophrologie ou d'autres techniques " actives " (c'est-à-dire demandant une implication de la parturiente) et ont, pour certaines d'entre elles, noté un recours moindre aux antalgiques ou aux demandes de péridurale mais les résultats en sont souvent biaisés par l'absence de tirage au sort et la difficulté de comparer les populations puisqu'il s'agit de patientes adhérant à cette technique. 
Bref, si la préparation à la naissance reste un outil indispensable d'accompagnement, un espace de parole ou un lieu d'échange et un besoin pour beaucoup de mamans enceintes, son rôle sur l'intensité ressentie des douleurs reste à prouver scientifiquement.

Dans la mesure où, à l'heure actuelle, une majorité de Françaises accouchent sous péridurale, cette préparation tend à prendre une place un peu différente dans l'accompagnement des parturientes, on est passé en quelques années de l'impréparation à une préparation militaire, puis à la préparation magistrale pour aller vers une préparation physique suivie d'une préparation à la carte. Celle-ci est plus mode, proche du coaching dont on parle beaucoup et va utiliser des méthodes ayant rarement apporté la preuve de leur utilité, mais donnant l'impression d'être davantage actrice et donc sus- ceptibles d'améliorer le vécu de la naissance. Elles ont l'avantage de laisser une grande possibilité de choix avec des préparations faisant appel aussi bien à la méthode classique individuelle qu'à I'haptonomie, la méthode Pilates, la sophrologie, la fasciathérapie, la préparation en piscine, le yoga, le chant prénatal, l'hypnose, la gymnastique prénatale (ou l'antigymnastique) et bien d'autres méthodes existantes ou restant à imaginer. 